\title{
The Jiangmen Underground Neutrino Observatory
}

\author{
Marco GRASSI ${ }^{* \dagger}$ \\ IHEP, Chinese Academy of Sciences, Beijing, P.R.China \\ E-mail: mgrassi@ihep.ac.cn \\ on behalf of the JUNO Collaboration
}

The Jiangmen Underground Neutrino Observatory (JUNO) is a large and high precision liquid scintillator detector under construction in the south of China. With its $20 \mathrm{kt}$ target mass, it aims to achieve an unprecedented 3\% energy resolution at $1 \mathrm{MeV}$. Its main goal is to study the disappearance of reactor antineutrino to determine the neutrino mass ordering, and to precisely measure the mixing parameters $\theta_{12}, \Delta m_{12}^{2}$, and $\Delta m_{e e}^{2}$. It also aims to detect neutrinos emitted from radioactive processes taking place within the inner layers of the Earth (geoneutrinos), as well as neutrinos produced during rare supernova bursts. Neutrinos emitted in solar nuclear reactions could also be observed, if stringent radiopurity requirements on the scintillator are met. This manuscript provides some highlights of JUNO's Physics Programme, and describes the detector design, as well as the ongoing detector R\&D.

XIII International Conference on Heavy Quarks and Leptons

22-27 May, 2016

Blacksburg, Virginia, USA

\footnotetext{
* Speaker.

${ }^{\dagger}$ MG acknowledges support from the Chinese Academy of Sciences President's International Fellowship Initiative grant 2015PM007.
} 


\section{Introduction}

The Jiangmen Underground Neutrino Observatory (JUNO) is a Liquid Scintillator Antineutrino Detector (LAND) currently under construction in the south of China (Jiangmen city, Guangdong province). Once completed, it will be the largest LAND ever built, consisting in a $20 \mathrm{kt}$ target mass made of Linear Alkyl-Benzene (LAB) liquid scintillator (LS), monitored by roughly 17000 twenty-inch high-quantum efficiency (QE) photomultipliers (PMTs) providing a 78\% photocoverage. Large photocoverage and large $\mathrm{QE}$ are two pivotal parameters of the experiment, which allow an unprecedented 3\% energy resolution at $1 \mathrm{MeV}$. The conceptual design report [1] foresees the LS to be contained in an acrylic sphere $12 \mathrm{~cm}$ thick and $35.4 \mathrm{~m}$ wide, and the whole detector to be immersed in a cylindrical water pool, acting both as a moderator for the environmental radioactivity, and as a Cherenkov detector to tag and veto cosmic muons. The ultimate control and minimal impact of calorimetry systematics is of maximal importance to achieve the aforementioned energy resolution. For this reason, a novel LAND design was introduced, where a second layer of small PMTs is used to provide a second calorimetry handle with complementary systematic budget, allowing a combined, more precise and accurate energy scale definition. This calorimetry redundancy system is still under optimisation considerations in the context of JUNO physics.

JUNO's main physics goal is to study neutrino oscillations by detecting reactor $\bar{v}_{e}$ produced by two nuclear power plants, both $53 \mathrm{~km}$ distant from the detector, with a total nominal power of $36 \mathrm{GW}_{\text {th }}$ ( $75 \%$ of which is scheduled to be available at the beginning of the data taking). JUNO will be the first LAND to observe simultaneously both solar and atmospheric oscillations, and it aims to determine the neutrino mass ordering through their interference. JUNO is also expected to measure several PMNS oscillation parameters [2] with a precision better than $1 \%$. Moreover, JUNO has a rich physics programme focused on neutrinos not originating from reactors. For the sake of brevity, here we review neutrinos from supernova (SN) burst, solar neutrinos, and geoneutrinos, but a complete description of JUNO's physics goals can be found in [3].

\section{Neutrino Physics at JUNO}

\section{REACTOR NEUTRINOS}

Nuclear power plants are pure, powerful and isotropic sources of $\bar{v}_{e}$. Recent and past experiments located at different baselines with respect to nuclear power plants successfully exploited the detection of $\bar{v}_{\mathrm{e}}$ to prove neutrino oscillation, and to precisely measure some of the oscillation parameters $[4,5,6,7]$. JUNO aims to detect $\bar{v}_{\mathrm{e}}$ coming from two of the most powerful nuclear power plants in the world - Taishan and Yiangjiang, located in the Chinese province of Guangdongto perform a precise measurement of their survival probability as a function of their energy. $\bar{v}_{\mathrm{e}}$ detection takes place through the Inverse Beta Decay (IBD) process, where the $\bar{v}_{\mathrm{e}}$ interacts with a proton of the liquid scintillator yielding a positron and a neutron, namely

$$
\bar{v}_{e}+p \rightarrow e^{+}+n
$$

The positron loses all its energy via ionisation, and eventually annihilates. Such energy deposition happens instantaneously, i.e. in a timescale much shorter than the typical LS deexcitation, and is called prompt. On the contrary, the neutron loses its energy via thermal interactions, and eventually 


\begin{tabular}{|c|c|c|c|c|c|c|c|}
\hline Selection & IBD efficiency & IBD & Geo- $v \mathrm{~s}$ & Accidental & ${ }^{9} \mathrm{Li} /{ }^{8} \mathrm{He}$ & Fast $n$ & $(\alpha, n)$ \\
\hline- & - & 83 & 1.5 & $\sim 5.7 \times 10^{4}$ & 84 & - & - \\
\hline Fiducial volume & $91.8 \%$ & 76 & 1.4 & \multirow{3}{*}{410} & 77 & \multirow[t]{5}{*}{0.1} & \multirow[t]{5}{*}{0.05} \\
\hline Energy cut & $97.8 \%$ & \multirow{3}{*}{73} & \multirow{3}{*}{1.3} & & \multirow{3}{*}{71} & & \\
\hline Time cut & $99.1 \%$ & & & & & & \\
\hline Vertex cut & $98.7 \%$ & & & 1.1 & & & \\
\hline Muon veto & $83 \%$ & 60 & 1.1 & 0.9 & 1.6 & & \\
\hline Combined & $73 \%$ & 60 & \multicolumn{5}{|c|}{3.8} \\
\hline
\end{tabular}

Table 1: Signal and backgrounds daily rates, together with the efficiencies of the antineutrino selection criteria. From [3].

gets captured by an hydrogen of the LS with a $\tau \sim 200 \mu$ s (delayed energy deposition), yielding a 2.2 MeV gamma. Because of the mass difference between the neutron and the positron, the latter retains most of the $\bar{v}_{\mathrm{e}}$ momentum, and the prompt energy is an accurate proxy for the $\bar{v}_{\mathrm{e}}$ energy. The delayed energy deposition is a unique signal signature, and allows a powerful background rejection by means of a time and vertex correlation with the prompt energy deposition.

Natural radioactivity makes up for the largest background contribution. Most of it originates from the PMT glass and from the outer wall of the detector, and is effectively reduced by applying a $0.5 \mathrm{~m}$ fiducial volume cut. Residual natural radioactivity events might occasionally fall within the time and vertex windows used for signal selection, yielding accidental coincidences which are an irreducible background. $\alpha$ radioactivity resulting in neutrons being ejected from stable nuclei is counted separately, and is referred to as $(\alpha, n)$. Geoneutrinos are considered a background from the point of view of reactor physics, and need to be properly subtracted. The remaining backgrounds originate from the spallation of cosmogenic muons on LS molecules. They comprise long lived isotopes $\left({ }^{9} \mathrm{Li}\right.$ and $\left.{ }^{8} \mathrm{He}\right)$ decaying $\beta-n$, and fast neutrons. Signal and background daily rates are summarised in Table 1.

The black line in Fig. 1 shows the expected prompt energy spectrum. It features two distinct oscillation patterns, a slow one arising from the solar mass splitting, and a fast one arising from the atmospheric mass splitting. The neutrino mass ordering information is embedded in the interference between the two oscillation modes, and can be extracted using either a Fourier-based or a $\chi^{2}$-based analysis. In the following we consider the latter (a description of the former can be found in [8]), and we quantify the sensitivity to the mass ordering as the $\Delta \chi^{2}$ obtained from fitting the prompt energy spectrum under the two hypotheses of normal and inverted mass ordering:

$$
\Delta \chi^{2}=\mid \chi_{\min }^{2}(\text { normal })-\chi_{\text {min }}^{2}(\text { inverted }) \mid .
$$

Such a fit strongly relies on the capability to resolve the fast oscillation pattern, which in turn depends on both detector energy resolution and statistics. Fig. 2 shows $\Delta \chi^{2}$ contours under different assumptions of these parameters. Six years of data with an energy resolution of $3 \%$ at $1 \mathrm{MeV}$ allow to reach $\Delta \chi^{2}=10$ by fitting JUNO's data alone. If we further constrain the fit using a prospective $1.5 \%$ determination of $\Delta_{\mu \mu}$ at MiNOs [10], $\Delta \chi^{2}$ increases up to 14 .

The four neutrino mixing parameters relevant to describe the JUNO energy spectrum are $\theta_{13}$, $\theta_{12}, \Delta m_{12}^{2}$, and $\Delta m_{e e}^{2}$ (a definition of $\Delta m_{e e}^{2}$ can be found in the supplement material of [5]). JUNO 
won't be able to outperform Daya Bay in the determination of $\theta_{13}$ since its baseline is optimised to be at the solar oscillation maximum, but it is expected to carry out a measurement of the remaining three parameters with a sub-percent precision. A detailed $\chi^{2}$-based sensitivity analysis shows that, after considering shape and normalisation uncertainties due to both background and detector response, six years of data allow to determine $\theta_{12}, \Delta m_{12}^{2}$, and $\Delta m_{e e}^{2}$ with a precision of $0.67 \%$, $0.59 \%, 0.44 \%$ respectively. It is worth stressing that the determination of the solar parameters does not depend on the energy resolution, because the solar oscillation manifests itself through broad features in energy spectrum. As a consequence, such measurement can be performed even while the detector response is still being commissioned —early stage of the experiment - and, more importantly, can also be performed with the large- and small-PMT systems independently (the latter features a $\sim 10 \%$ energy resolution), allowing a powerful validation of the solar parameters determination.

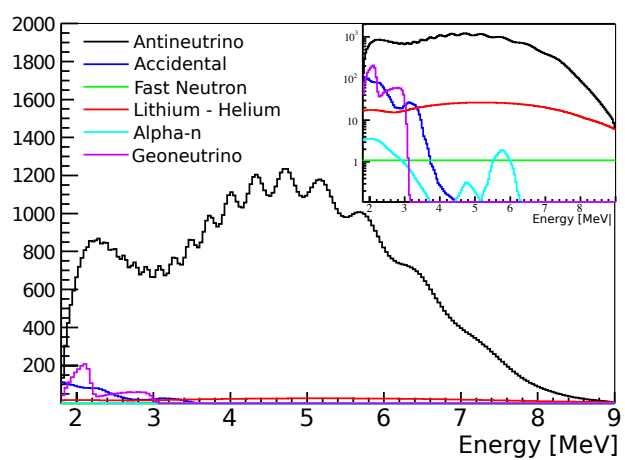

Figure 1. Energy spectra of the antineutrino signal and of the main background sources: accidental coincidences, cosmogenic ${ }^{8} \mathrm{He}$ and ${ }^{9} \mathrm{Li}$, fast neutrons, ${ }^{13} \mathrm{C}(\alpha, n){ }^{16} \mathrm{O}$, and geoneutrinos. From [3].

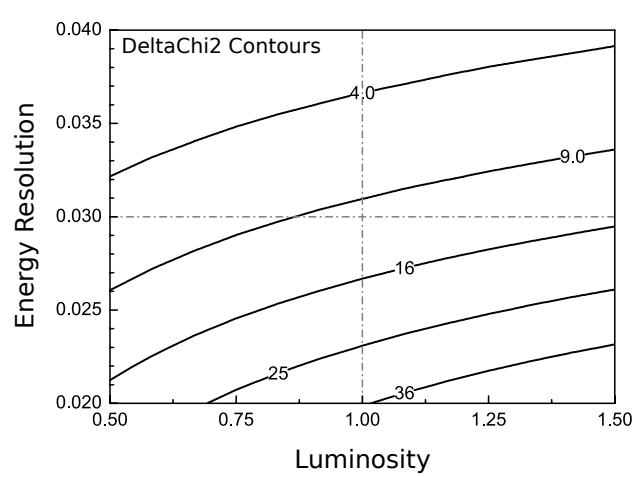

Figure 2. JUNO's sensitivity to the mass ordering determination as a function of the event statistics (luminosity) and of the detector energy resolution. Solid lines represent iso- $\Delta \chi^{2}$ contours. Dashed lines show the nominal runtime of six years (including $80 \%$ efficiency) and the nominal 3\% energy resolution. From [3].

\section{SUPERNOVA BURST NeUTRINOS}

A supernova $\mathrm{SN}$ is a stellar explosion that briefly outshines an entire galaxy, radiating as much energy as the Sun or any ordinary star is expected to emit over its entire life span. During such explosion, $99 \%$ of the gravitational binding energy of the newly formed neutron star is emitted in the form of neutrinos. The observation of SN neutrinos is expected to play a relevant role both in particle physics and astrophysics. Here we focus on the latter, where a SN signal might help answering several fundamental questions, such as (I) what are the conditions inside massive stars during their evolution? (II) what mechanism triggers the SN explosion? (III) are SN explosions responsible for the production of heavy chemical elements? and (IV) is the compact remnant a neutron star or a black hole? Each of these questions would deserve a dedicated section, but because of the limited space we consider (I) as a case study.

The Standard Stellar Evolution Model describes temperature and density of a star as a function of time and distance from its centre. Optical observations usually provide benchmark data to test 
it, but they have little power in constraining the model of the star's innermost layers. Indeed, the star's high density results in optical photons propagating mainly via diffusion, hence losing all the information about the stellar core. On the contrary, neutrinos interact weakly with stellar matter, and they represent a powerful tool to probe the inner structure of the star. For a star close to its collapse, neutrino production is dominated by thermal processes (mainly $e^{+}-e^{-}$annihilating into $v-\bar{v}$ pairs). That is, the neutrino production rate, and the neutrino mean energy, both increase significantly with temperature. As a result, the last stages of the star's nuclear burning produce the most abundant neutrino signal (called pre-SN $v$ ), easier to detect and powerful in describing the stellar evolution.

Fig. 3 shows the simulated inverse beta decay (IBD) event rate in JUNO for the nearest possible $\mathrm{SN}$ progenitor (the red supergiant Betelgeuse) whose mass is taken to be 20 solar masses $\left(\mathrm{M}_{\odot}\right)$ at a distance of $0.2 \mathrm{kpc}$. The sudden drop in the rate around 0.6 day before the $\mathrm{SN}$ explosion is ascribable to a drop of the core temperature, mostly due to the silicon depletion of the core itself. In the case of a SN explosion, JUNO's capability to precisely measure the position of such a dip could serve as a discriminator for different progenitor star masses. Moreover, the quick rise starting few hours prior to core collapse makes JUNO an ultimate pre-warning system of SN explosion, extremely valuable to the astrophysics community.

For a typical galactic SN at $10 \mathrm{kpc}$, there will be more than 5000 signal events solely from the IBD channel. However, several other neutrino interactions contribute to the total event rate. They differ in terms of total yield, energy spectrum and energy threshold. Fig. 4 shows all of them together, where $\left(E_{d}\right)$ is the deposited visible energy in the detector, $\left(E^{\text {th }}\right)$ is the energy threshold of each process, $(v-\mathrm{p})$ are the neutral current interactions on protons, $(v$-e $)$ are the elastic scatterings on electrons, $\left({ }^{12} \mathrm{C} \mathrm{NC}\right)$ are the neutral-current-mediated carbon excitations, $\left({ }^{12} \mathrm{~N} \mathrm{CC}\right)$ are the charged current $v_{\mathrm{e}}$ interactions on ${ }^{12} \mathrm{C}$, and $\left({ }^{12} \mathrm{~B} \mathrm{CC}\right)$ are the the same charged current interactions initiated by $\bar{v}_{\mathrm{e}}$.

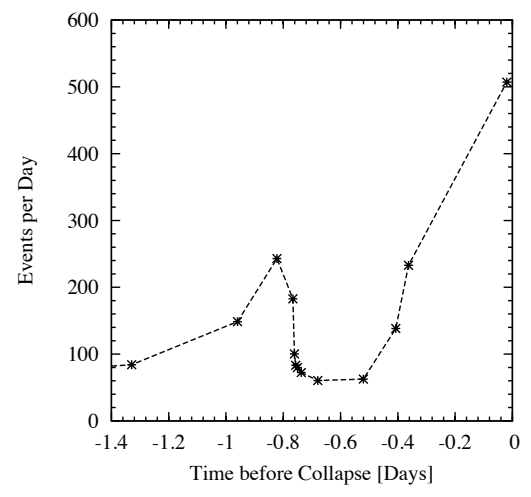

Figure 3. Neutrino event rate in JUNO for a massive star $\left(20 \mathrm{M}_{\odot}\right)$ distant $0.2 \mathrm{kpc}$ from the Earth, the same as the nearest possible SN progenitor Betelgeuse. From [3].

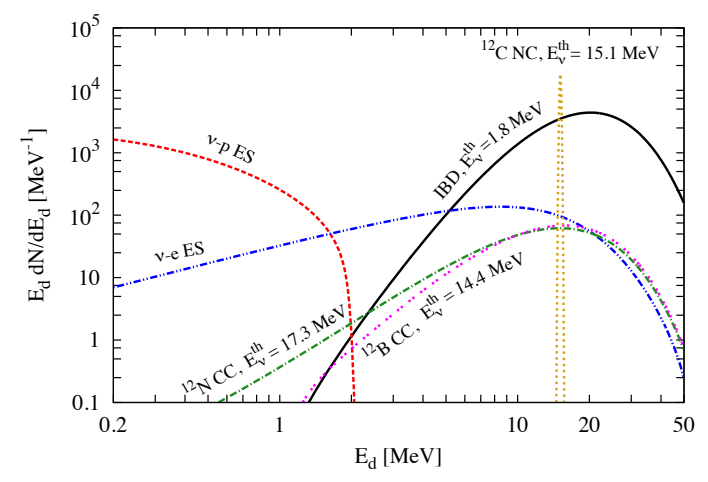

Figure 4. Neutrino energy spectra in the JUNO detector for a $\mathrm{SN}$ at $10 \mathrm{kpc}$, where no neutrino flavor conversions is assumed. $E_{d}$ is the visible energy, and $E^{\text {th }}$ the threshold energy. From [3].

\section{Solar NeUtrinos}

The Sun is a powerful source of $v_{\mathrm{e}}$ with $\mathrm{O}(1 \mathrm{MeV})$ energy, produced in the thermonuclear fusion reactions happening in the solar core. JUNO's solar neutrino programme focuses on those 
emitted by the ${ }^{7} \mathrm{Be}$ and ${ }^{8} \mathrm{~B}$ chains. Indeed, despite the great achievements of the last decades, there are still important aspects of solar neutrino physics to clarify, and some questions of great relevance for astrophysics and elementary particle physics waiting for definite solutions. Two of the most important ones are (I) the solution of the solar metallicity problem, and (II) the detailed analysis of the oscillation-probability energy dependence in the lower end of the ${ }^{8} \mathrm{~B}$ neutrino spectrum.

(I) The solar metallicity problem emerged when the former agreement between Standard Solar Model (SSM) and solar data got compromised by the revision of the solar surface heavy element content, leading to a discrepancy between the SSM and helioseismology results. The predictions of different SSM versions differ (also) by the ${ }^{8} \mathrm{~B}$ and ${ }^{7} \mathrm{Be}$ neutrino fluxes. JUNO's capability to determine these fluxes with high accuracy, together with data (coming from other future experiments) about the CNO fluxes, could help solving this key issue in nuclear astrophysics.

(II) According to the Mikheyev-Smirnov-Wolfenstein (MSW) effect, neutrino oscillation parameters are different if a neutrino propagates through matter or in vacuum. In the case of solar $v_{\mathrm{e}}$, the transition between the two behaviors is expected to happen in the $1 \sim 3 \mathrm{MeV}$ energy range, therefore solar ${ }^{8} \mathrm{~B}$ neutrinos — with their continuous energy spectrum stretching far beyond $3 \mathrm{MeV}$ — are a privileged tool to study the MSW-modulated energy dependence. The theory predicts a smooth transition between the vacuum and matter related $v_{\mathrm{e}}$-survival probabilities, namely an upturn in the spectrum. However, none of the existing experiments so far observed a clear evidence of this effect. The only exception is Super-Kamiokande, which got a mild evidence of the up-turn in its data [9]. JUNO's capability to perform an independent and high-significance test of the up-turn existence would be extremely important to confirm the consistency of the standard LMA-MSW solution, or to indicate any possible deviations from this standard paradigm.

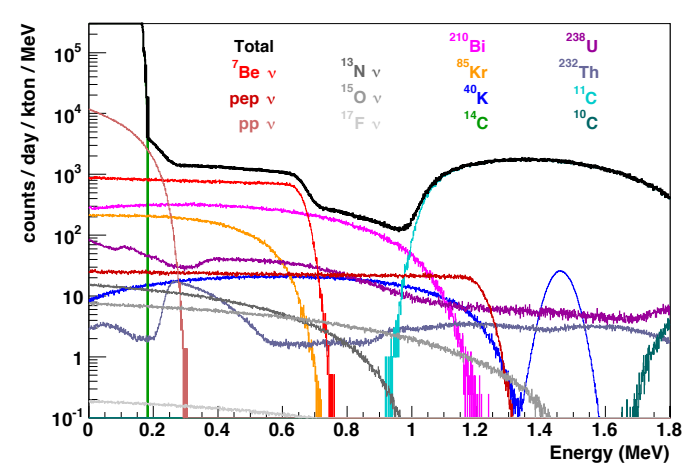

Figure 5. Energy spectra of singles from natural radioactivity (background) in the ideal radiopurity scenario, together with the ${ }^{7} \mathrm{Be}$ neutrino signal. From [3].

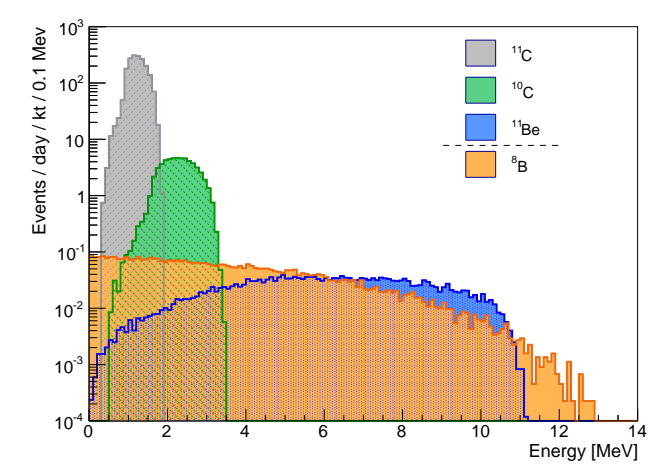

Figure 6. Energy spectra of the ${ }^{8} \mathrm{~B}$ neutrino signal and of the cosmogenic backgrounds. From [3].

The challenge in detecting solar $v_{\mathrm{e}}$ at JUNO is that they are detected only by elastic scattering, which results in an experimental signature (single energy deposition) indistinguishable from most of the background processes. The two main background sources are natural radioactivity and cosmogenic isotopes. The first needs to be suppressed by achieving a high-level radiopurity in all the detector components. JUNO's baseline radiopurity scheme envisages a ${ }^{232} \mathrm{Th},{ }^{40} \mathrm{~K},{ }^{14} \mathrm{C}$ residual contamination at the level of $10^{-16}, 10^{-16}, 10^{-17} \mathrm{~g} / \mathrm{g}$ respectively. As a comparison, the same level 
of radiopurity was achieved during KamLAND solar phase, and it would allow JUNO to achieve a signal/background ratio of $1 / 3$. A more demanding radiopurity scheme (called ideal) requires the previous contamination levels to improve by one order of magnitude, which would correspond to Borexino phase I, and would allow a $2 / 1$ signal/background ratio. The energy spectra of the radioactive background processes in the case of ideal radiopurity, together with the signal ${ }^{7} \mathrm{Be} v_{\mathrm{e}}$, are shown in Fig. 5. Among the cosmogenic isotopes, the most dangerous are the long-lived ${ }^{11} \mathrm{C}$ $(\tau=24.4 \mathrm{~min}),{ }^{10} \mathrm{C}(\tau=27.8 \mathrm{~s})$, and ${ }^{11} \mathrm{Be}(\tau=19.9 \mathrm{~s})$, since they cannot be suppressed by a muon veto without introducing large deadtime. The energy spectra of these background events are shown in Fig. 6. The only way to handle them is to tag them via a three-fold coincidence (muon + spallation neutron + isotope decay) and subtract them statistically from the total spectrum.

\section{GEOEUTRINOS}

Over the last half a century, the Earth's surface heat flow has been established to be ( $46 \pm$ 3) TW [11]. However the community is still vigorously debating what fraction of this power comes from primordial versus radioactive sources. This debate touches on the composition of the Earth, the question of chemical layering in the mantle, the nature of mantle convection, the energy needed to drive plate tectonics, and the power source of the geodynamo, which powers the magnetosphere that shields the Earth from the harmful cosmic ray flux. Radioactive beta-decays of heavy elements (such as Th and U) taking place inside the Earth result in an upwards $\bar{v}_{\mathrm{e}}$ flux (also called geoneutrino flux) which can be detected at JUNO by means of IBD reactions. A precise measurement of such flux would allow us to accurately define the absolute abundance of Th and $U$ in the Earth, which in turn would allow us to: (I) define the building blocks, the chondritic meteorites, that formed the Earth, (II) discriminate models of parameterised mantle convection that define the thermal evolution of the Earth, (III) potentially identify and characterize deep, hidden reservoirs in the mantle, and (IV) fix the radiogenic contribution to the terrestrial heat flow. Moreover, such studies can place stringent limits on the power of any natural nuclear reactor in or near the Earth's core.

The main experimental challenge in detecting a geoneutrino signal is to disentangle it from the reactor $\bar{v}_{\mathrm{e}}$ signal, which is overwhelming. Such a separation can be done only via statistical subtraction, and it relies heavily on a precise modeling of the low-energy reactor $\bar{v}_{\mathrm{e}}$ spectrum. Moreover, to interpret the geoneutrino signal in terms of mantle's radioactivity, the contribution from the Earth's crust need to be subtracted, since it has been well established that the crust surrounding the detector will play a major role in total geoneutrino budget. Thus, to understand the relative contributions from the crust and mantle to the total geoneutrino signal at JUNO, detailed geological, geochemical, and geophysical studies need to be performed in the areas surrounding the detector.

\section{The JUNO Detector}

The JUNO detector is designed to be placed $720 \mathrm{~m}$ underground and comprises several components. We refer to the Central Detector as the $35.4 \mathrm{~m}$ wide acrylic sphere containing $20 \mathrm{kt}$ of purified LAB scintillator (target volume). The target volume is monitored by 1700020 -inch PMTs and 34000 3-inch PMTs, installed on a Stainless Steel Lattice Shell surrounding the acrylic sphere 
at a distance of few meters. The overall photocathode density is the largest ever built, and accounts for an unprecedented $78 \%$ photocoverage, yielding 1200 photoelectrons/MeV, pivotal to achieve the $3 \%$ energy resolution at $1 \mathrm{MeV}$. The set of large PMTs in unevenly split between dynode-based Hamamatsu Photonics PMTs, and Micro Channel Plate-based PMTs produced by the Chinese North Night Vision Technology, the latter accounting for three fourth of the total. The whole Central Detector is immersed in a cylindrical water pool filled with ultra-pure water. Since the water penetrates the Lattice Shell supporting the PMTs, it acts as a buffer shielding the target volume from natural radioactivity arising from the rock and from the PMT glass. The water pool is instrumented with 2000 20-inch PMTs to detect Cherenkov light, hence acting as an active muon veto. To better track cosmic muons, a tracker consisting of three layer of plastic scintillator (inherited from the OPERA detector [12]) is deployed on top of the water pool, covering $~ 50 \%$ of the water pool surface. The chimney connecting the acrylic sphere to the surface of the water pool is also instrumented in order to detect stopping muons that might generate untagged background events.

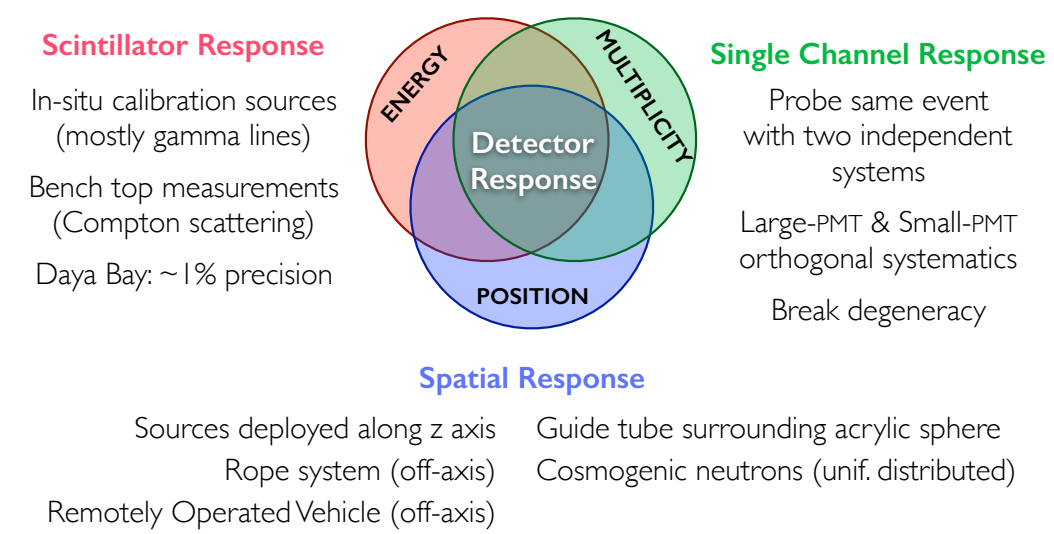

Figure 7. Summary of the methods put in place to understand the detector response.

The unprecedented light level of 1200 photoelectrons/MeV allows us to achieve a stochastic resolution term smaller than $2.9 \% / \sqrt{E}$. However, the design resolution of $3 \%$ at $1 \mathrm{MeV}$ requires to keep non-stochastic components of the energy resolution below $1 \%$. These components mostly arise from an imperfect knowledge of the detector response, and can be grouped into energy-related, multiplicity-related and position-related (see Fig. 7). The latter are addressed by scanning the detector with four calibration systems: (I) one along the $\mathrm{z}$ axis, (II) one based on ropes deployed from the chimney and able to reach most of the off-axis region, (III) one based on a guide-tube running along the acrylic vessel to study the outermost shell of the target volume, and (IV) one based on a Remote-Operated Vehicle to check eventual blind spots. The energy-related uncertainties are addressed by deploying radioactive sources yielding gamma lines at different energies and neutrons, as well as studying the LS response with bench-top experiments. Finally, the multiplicity-related uncertainties are addressed by means of the small-PMT system, where the size of the PMTs ensures that each phototube works in photon-counting mode -i.e. it detects one photoelectron at most-, effectively resulting in a digital calorimetry estimator. This estimator is designed to be much more robust to uncertainties typical of large phototubes, such as low-resilience to magnetic field, uniformity of the photocathode deposition, knowledge of the collection efficiency profile, 
pileup of multiple hits, complex waveform reconstruction, and saturation of the output signal. The small-PMT system further provides a powerful handle to help break the degeneracy existing among the three groups, namely the cross-talk existing among different systematic uncertainties. As an example, an imperfect reconstruction of those PMT waveforms experiencing large pile-up might easily mimic a residual detector non-uniformity. Such degeneracy could likely become the main limitation to a full understanding of the detector response. The study of calibration events with both the large- and the small-PMT systems, each of them providing an independent energy measurement characterised by its own hardware and reconstruction method, allows us to sample the same energy deposition with effectively two detectors, each one experiencing a completely different systematic uncertainty budget. The comparison and the cross-calibration of these two systems is therefore geared to become a valuable asset in understanding and minimising the non-stochastic resolution terms.

\section{Conclusions}

The Jiangmen Underground Neutrino Observatory (JUNO) is a large and high precision liquid scintillator detector under construction in the south of China. With its $20 \mathrm{kt}$ target mass, it aims to achieve an unprecedented 3\% energy resolution at $1 \mathrm{MeV}$. To this end, a 1200 photoelectrons/ $\mathrm{MeV}$ light level is required, which drives the effort to reach a LS attenuation length larger than $20 \mathrm{~m}$, and a photocoverage larger than $75 \%$. Such stringent requirements are pivotal to determine the neutrino mass ordering through the analysis of $\bar{v}_{\mathrm{e}}$ produced by two powerful nuclear power plants $\left(36 \mathrm{GW}_{\text {th }}\right.$ nominal power) at a baseline of $53 \mathrm{~km}$. The investigation of reactor $\bar{v}_{\mathrm{e}}$ is also aimed to determine the neutrino mixing parameters $\theta_{12}, \Delta m_{12}^{2}$, and $\Delta m_{e e}^{2}$ with a precision better than $1 \%$. JUNO will further be able to detect neutrinos coming from a supernova burst, and geoneutrinos originating from the inner layers of our planet, hence extending JUNO's Physics Programme far beyond the reactor neutrino physics. Depending on the scintillator radiopurity, neutrinos originating from the Sun could also be detected, shedding light on the solar metallicity problem and on the MikheyevSmirnov-Wolfenstein turn on curve. JUNO's data taking is foreseen to begin in 2020.

\section{References}

[1] F. An et al. [JUNO Collaboration], JUNO Conceptual Design Report. [arXiv: 1508.07166]

[2] Z.Maki et al., Prog. Theor. Phys. 28 (1962) 870, B.Pontecorvo, Zh. Eksp. Theor. Fis., 53 (1967) 1717, V.Gribov and B.Pontecorvo, Phys. Lett. B 28 (1969) 493.

[3] F. An et al. [JUNO Collaboration], J. Phys. G 43 (2016) no.3, 030401 [arXiv:1507.05613].

[4] S. Abe et al. (KamLAND), Phys. Rev. Lett. 100 (2008) 221803, [arXiv:0801.4589].

[5] F. P. An et al. (Daya Bay), Phys. Rev. Lett. 115 (2015) 111802, [arXiv:1505.03456].

[6] Y. Abe et al. (Double Chooz), JHEP 01 (2016) 163, [arXiv:1510.08937].

[7] J.H. Choi et al. (RENO), Phys.Rev.Lett. 116 (2016) 211801, [arXiv:1511.05849].

[8] L. Zhan, Y. Wang, J. Cao and L. Wen, Phys. Rev. D 79 (2009) 073007 [arXiv:0901.2976].

[9] A. Renshaw et al. [Super-Kamiokande Collaboration], Phys. Rev. Lett. 112 (2014) 9, 091805. 
[10] P. Adamson et al. (MINOS), Phys.Rev.Lett. 112 (2014) 191801, [arXiv:1403.0867].

[11] J. H. Davies and D. R. Davies, Solid Earth 1 (2010) 5.

[12] R. Acquafredda et al. (OPERA), JINST 4 (2009) P04018. 